\title{
Road Occupancy Prediction of Traffic Participants
}

\author{
Matthias Althoff, Daniel Heß, and Florian Gambert
}

\begin{abstract}
We predict the road occupancy of traffic participants for collision avoidance systems. The occupancy sets are computed for consecutive time intervals and contain all reachable positions of traffic participants in compliance with a proposed dynamic model. Those sets make it possible to check if planned emergency maneuvers are collision-free in all possible future scenarios. However, no algorithm exists for exactly computing the occupancy when the model forbids unrealistic behavior such as leaving road boundaries or largely exceeding speed limits. For this reason, we provide methods to tightly overapproximate occupancy sets to ensure safe emergency maneuvers. We demonstrate the applicability of the approach by numerical examples, which show that the occupancy computation is not only efficient, but also tight enough to trigger emergency maneuvers almost at the last possible point in time.
\end{abstract}

\section{INTRODUCTION}

Collision avoidance systems are a key contribution to safer vehicles [1]. We consider collision avoidance systems that completely take over the control of a vehicle when the human driver is about to miss the last chance to avoid a collision. To determine the last possible safe maneuver, the road occupancy of other traffic participants is predicted over time. Possible options to avoid occupancy sets of other traffic participants are generated by a trajectory planner [2]. In order to rigorously guarantee safety for certification purposes, the occupancy has to include all possible positions [3], which we refer to as overapproximative occupancy sets. The presented approach can be easily extended to collaborative emergency maneuvers using vehicle-to-vehicle communication since in this case the occupancies of collaborating traffic participants are known and thus a subset of the computed sets.

Exact occupancy sets can only be computed for rather simple dynamic models, such as point masses with constrained velocity or acceleration values [4], [5], or unicycle models with constant velocity [6]. For more complicated models considering road boundaries and maximum velocities, such as the one used in this work, there are no known approaches to exactly compute the occupancy set. In order to guarantee safety for more complicated models, we compute tight overapproximations of their occupancy sets while most other approaches compute non-overapproximative approximations. In [7], the occupancy of other traffic participants is computed heuristically to trigger collision probability computations when a collision is possible. By varying only the direction of acceleration, one obtains behavior sets as presented in [8].

M. Althoff is with the department of Computer Science and Automation, D. $\mathrm{He} B$ is with the department of Mechanical Engineering, F. Gambert is with the department of Electrical Engineering, Ilmenau University of Technology, 98693 Ilmenau, Germany. matthias.althoff, daniel.hess, florian.gambert\}etu-ilmenau.de
The need for computing overapproximative occupancy sets of other traffic participants is described in [9], but no specific computation scheme is presented in that work.

An alternative approach to determine whether a collisionfree maneuver exists is to compute a finite number of possible future behaviors of other traffic participants. However, this approach has several disadvantages: Computing many scenarios is time consuming and additionally requires to perform collision checks for each possible behavior. Further, numerical simulation is not a formal technique, i.e., even when no unsafe combination of behaviors is found, the scenario might still be unsafe since the problematic behavior might be missed. Finite sets of possible behaviors according to turning possibilities (left, right, go straight) within a road network are investigated in [10]. Predictions of a single behavior for each traffic participant are presented in [11]-[13]. Predictions of behavior patterns (such as lane change, left turn, etc.) are described in [14], [15]. Due to the importance of the prediction of lane changes, much work has focused on this problem [16]-[19]. Note that none of the previous works investigates lane changes in an overapproximative way as done in this work.

When the finite set of simulated behaviors is weighted by probabilities, which is referred to as Monte Carlo simulation, one obtains the probability for a collision [20], [21]. This is not the scope of this work, since we aim at computing evasive maneuvers that are guaranteed safe. Alternatives to Monte Carlo simulation are Markov chains [22], linearizations representing different operation modes in combination with Gaussian distributions [7], [23], Bayesian occupancy filters [24], and multiordered prediction models [25].

In Sec. II we summarize the basic idea. Sec. III presents the model that is used for computing the occupancy set in Sec. IV. Finally, the integration of the occupancy sets in a trajectory planner using rapidly exploring random trees is presented in Sec. V, followed by the conclusion in Sec. VI.

\section{BASIC IDEA}

The proposed collision avoidance system is based on a software module that constantly checks whether collisionfree maneuvers exist. No intervention takes place when collision-free maneuvers exist, but when only one or a limited set of safe maneuvers remains, the best option is automatically executed until the vehicle comes to a safe stop or the vehicle can be safely taken over by the driver. In order to determine safe maneuvers, it is required to predict the set of possible future positions of other traffic participants, which is referred to as their occupancy (see Fig. 1, (1). The planning algorithm generates possible maneuvers (see 
(1) occupancy prediction

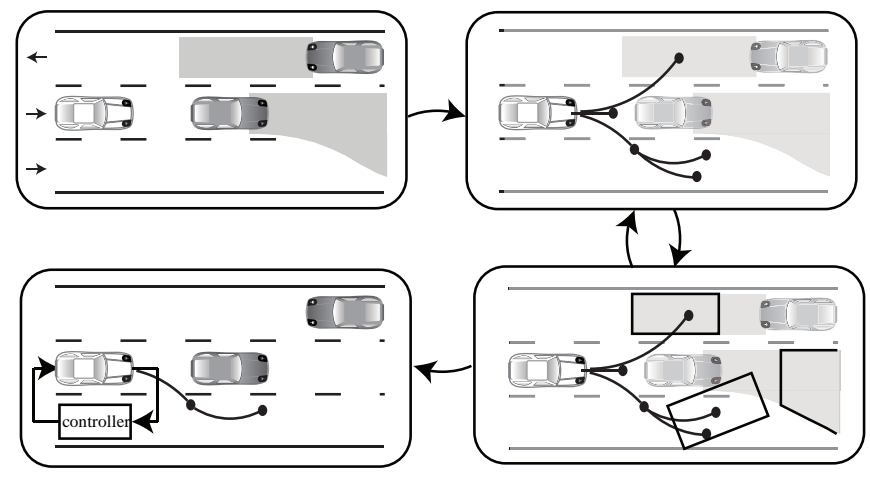

(4) trajectory tracking
(2) trajectory planning

(3) collision checking

Fig. 1. Collision avoidance concept.

Fig. 1, (2), which are then evaluated for collision avoidance by checking if the occupancy of the ego vehicle (i.e. the vehicle under consideration) intersects with the occupancy of any other traffic participants (see Fig. 1, (3). The collision check in Fig. 1 is performed for the occupied areas of short consecutive time intervals $\left[t_{k}, t_{k+1}\right]$. The regions for a single time interval are indicated by black lines in Fig. 1, (3), while the gray area shows the occupancy of the complete time horizon. We use consecutive time intervals instead of points in time to ensure that collisions are not missed in between points in time. When only a very limited set of safe maneuvers is found, the best emergency maneuver is executed using a trajectory tracking controller (see Fig. 1, (4)). Candidates for trajectory tracking controllers for emergency maneuvers are evaluated in a previous work [26].

This work focuses on the prediction of the occupancy of each traffic participant. We guarantee that each occupancy set encloses all possible positions in compliance with a proposed dynamic model and by considering measurement errors, uncertain parameters, and unknown driver behavior. In order to ensure that bounds on the aforementioned uncertainties enclose real data, one can safely overestimate values, or tune the approach towards collision mitigation by assuming small sets of uncertainties.

\section{VEHICLE MODEL}

This section presents the dynamic model used for predicting the occupancy of traffic participants. The model for this purpose is much simpler compared to models used for designing trajectory tracking controllers. One reason is that parameters of other traffic participants are typically unknown (unless they would be transmitted via vehicle-to-vehicle communication) so that complicated models requiring identified parameters are useless. The other reason is that the main source of uncertainty is the model input (changing lane, accelerating/decelerating) and not a potential inaccuracy of the dynamic model. We propose a model that satisfies the following constraints:

$C 1$ : positive longitudinal acceleration is stopped when a parameterized speed $v_{\max }$ is reached ( $v_{\max }$ could be set

to a certain percentage above the official speed limit). $C 2$ : driving backwards in a lane is not allowed.

$C 3$ : positive longitudinal acceleration is inversely proportional with speed above a parameterized speed $v_{S}$ (modeling a maximum engine power).

$C 4$ : maximum absolute acceleration is limited by $a_{\max }$.

$C 5$ : actions that cause leaving the road/lane boundary are forbidden.

The position $s$ and velocity $v$ is obtained by integration of the acceleration $a$, where components in $x$ - and $y$-direction are indicated by the corresponding subscripts. In order to write the dynamics in state space form, we introduce the states $x_{1}=s_{x}, x_{2}=s_{y}, x_{3}=v_{x}$, and $x_{4}=v_{y}$ :

$\dot{x}(t)=A x(t)+u(t), A=\left[\begin{array}{llll}0 & 0 & 1 & 0 \\ 0 & 0 & 0 & 1 \\ 0 & 0 & 0 & 0 \\ 0 & 0 & 0 & 0\end{array}\right], u(t)=\left[\begin{array}{c}0 \\ 0 \\ a_{x}(t) \\ a_{y}(t)\end{array}\right]$

In order to restrict $a_{x}(t)$ and $a_{y}(t)$ according to the constraints $C 1-C 5$, we introduce unit vectors that point towards the longitudinal and lateral direction of the vehicle: $\Phi(t)^{\text {long }}=\frac{1}{v}\left[v_{x}(t), v_{y}(t)\right]^{T}, \Phi(t)^{\text {lat }}=\frac{1}{v}\left[-v_{y}(t), v_{x}(t)\right]^{T}$, where $v=\left\|\left[v_{x}, v_{y}\right]^{T}\right\|_{2}$. This makes it possible to formulate $a_{x}, a_{y}$ by the longitudinal acceleration $a^{\text {long }}(t)$ and the lateral acceleration $a^{\text {lat }}(t)$ :

$$
\left[\begin{array}{l}
a_{x} \\
a_{y}
\end{array}\right]=\Phi^{\text {long }} a^{\text {long }}+\Phi^{\text {lat }} a^{\text {lat }}
$$

The lateral acceleration is determined by the maximum absolute acceleration and a normalized steering input $u_{1}$, where $u_{1}= \pm 1$ represents steering to the left or right using the full tire friction potential:

$$
a^{\text {lat }}=a_{\max } u_{1} .
$$

In order to consider constraint $C 4$, the remaining acceleration potential in longitudinal direction is limited to

$$
a_{\mathrm{c} 1}^{\text {long }}=\sqrt{a_{\max }^{2}-a^{\text {lat }^{2}}}
$$

The maximum longitudinal acceleration for the engine power $P$ and the vehicle mass $m$ is $\frac{P}{m v}=a_{\max } \frac{v_{S}}{v}$, where $v_{S}=\frac{P}{a_{\max } m}$ is the speed above which the acceleration is limited by the engine power and no longer by the tire friction. In case the parameter cannot be estimated, one can set $v_{S}=\infty$, which provides an overapproximation of the occupancy set. Similarly to the lateral acceleration, we introduce a normalized control input $u_{2}$ for the longitudinal acceleration, where $u_{2}= \pm 1$ represents full braking and full acceleration within the acceleration potential. Limited engine power, the restriction to forward driving, and the maximum speed (constraints $C 1-C 3$ ) are considered by limiting the acceleration to

$$
a_{\mathrm{c} 2}^{\text {long }}= \begin{cases}a_{\max } \frac{v_{S}}{v}, & v_{S}<v<v_{\max } \wedge u_{2}>0 \\ a_{\max }, & \left(0<v \leq v_{S} \vee\left(v>v_{S} \wedge u_{2} \leq 0\right)\right) \\ & \wedge v<v_{\max } \\ 0, & v \leq 0 \vee v \geq v_{\max }\end{cases}
$$


Combining $a_{\mathrm{c} 1}^{\text {long }}$ and $a_{\mathrm{c} 2}^{\text {long }}$ results in the longitudinal acceleration complying to constraints $C 1-C 4$ ( $C 5$ for road departure is considered later):

$$
a^{\text {long }}= \begin{cases}a_{\mathrm{c} 2}^{\text {long }} u_{2}, & a_{\mathrm{c} 2}^{\text {long }} u_{2} \leq a_{\mathrm{c} 1}^{\text {long }} \\ a_{\mathrm{c} 1}^{\text {long }}, & a_{\mathrm{c} 2}^{\text {long }} u_{2}>a_{\mathrm{c} 1}^{\text {long }}\end{cases}
$$

The method for computing the occupancy sets based on this model is presented in the next section.

\section{OCCUPANCY COMPUTATION}

Computing the occupancy of other traffic participants is challenging for several reasons. First, the set of initial states is uncertain due to measurement uncertainties. Second, one has to consider all behaviors for arbitrarily varying normalized inputs $u(t)=\left[\begin{array}{ll}u_{1}(t) & u_{2}(t)\end{array}\right]^{T}\left(u_{1}\right.$ : normalized steering, $u_{2}$ : normalized acceleration). Third, one has to restrict the behaviors to those that would not result in leaving the road boundary.

As previously discussed, simulation techniques are not capable of computing all possible behaviors when $x(0) \in \mathcal{R}(0)$ and $\forall t: u(t) \in \mathcal{U}$, where $\mathcal{R}(0)$ is the set of initial states and $\mathcal{U}$ is the two-dimensional unit box bounding the normalized input $u(t)$. However, all behaviors can be considered using reachability analysis (see e.g. [27]). After denoting a solution of the dynamic model proposed in Sec. III by $\chi(t, x(0), u(\cdot))$, where $u(\cdot)$ is the input trajectory, we define the reachable set as $\mathcal{R}(t)=$ $\{\chi(t, x(0), u(\cdot)) \mid x(0) \in \mathcal{R}(0), \forall \tau \in[0, t]: u(\tau) \in \mathcal{U}\}$. The occupancy would be obtained by projecting the states on the first and second coordinate, which are the positions $s_{x}$ and $s_{y}$.

Since the proposed dynamics is hybrid (mixed discrete and continuous) due to the switching of acceleration limits, a reachability analysis is infeasible considering the computation time restrictions of this application. For this reason, we compute different occupancy sets for different abstractions of the dynamic model. We show that intersecting those sets returns an overapproximation of the exact occupancy, which is formalized by introducing the projection operator $\operatorname{proj}()$ of a set and an operator reach() returning the reachable set of a model $M_{i}$.

Proposition 1 (Overapproximative Occupancy): Given are models $M_{i}, i=1 \ldots m$ which are abstractions of model $M_{0}$, i.e., reach $\left(M_{0}\right) \subseteq \operatorname{reach}\left(M_{i}\right)$. The occupancy of the model $M_{0}$ can be overapproximated by

$$
\operatorname{proj}\left(\operatorname{reach}\left(M_{0}\right)\right) \subseteq \bigcap_{i=1}^{m} \operatorname{proj}\left(\operatorname{reach}\left(M_{i}\right)\right) .
$$

Proof: Since $\operatorname{reach}\left(M_{0}\right) \subseteq \operatorname{reach}\left(M_{i}\right)$, we have that

$$
\begin{aligned}
\operatorname{reach}\left(M_{0}\right) & \subseteq \bigcap_{i=1}^{m} \operatorname{reach}\left(M_{i}\right) \\
\rightarrow \operatorname{proj}\left(\operatorname{reach}\left(M_{0}\right)\right) & \subseteq \operatorname{proj}\left(\bigcap_{i=1}^{m} \operatorname{reach}\left(M_{i}\right)\right)
\end{aligned}
$$

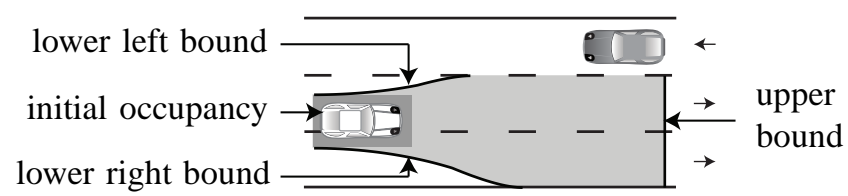

Fig. 2. Initial occupancy and boundaries of the occupancy set for a long time interval.

Further, it is shown in [28, Prop. 1] that

$$
\operatorname{proj}\left(\bigcap_{i=1}^{m} \operatorname{reach}\left(M_{i}\right)\right) \subseteq \bigcap_{i=1}^{m} \operatorname{proj}\left(\operatorname{reach}\left(M_{i}\right)\right),
$$

which proves the proposition.

Prop. 1 is applied for computing the occupancy of different abstractions of the real model, which are intersected to obtain a tight overapproximation. This approach is much faster compared to reachability analysis for the road occupancy computation and returns only small overapproximations. The first abstraction allows the vehicle to move arbitrarily in lateral direction, but considers the longitudinal dynamics along a path (see Sec. IV-A). The second abstraction considers limited absolute acceleration, but neglects constraints in the longitudinal dynamics, such as the maximum speed (see Sec. IV-B). Sec. IV-A provides the upper bound of the occupancy set in driving direction, while Sec. IV-B provides the lower left, and lower right bound, see Fig. 2.

\section{A. Occupancy Along Road Boundaries}

In this subsection, we use the abstraction that vehicles move along paths while considering constraints $C 1-C 4$, where the lateral positions are arbitrary within lane boundaries (see Fig. 3). The considered paths are centers of lanes, where the position along a path is specified by the path coordinate $s$. The path coordinate uniquely provides the $\mathrm{x}$ - and $\mathrm{y}$-position as well as the curvature radius by the functions $\left[s_{x}, s_{y}\right]^{T}=p(s)$ and $R=\frac{1}{\kappa(s)}$, respectively. The goal of this abstraction is to obtain the position along a path furthest away from the initial position. Because of the restricted movement along a path, the lateral acceleration is determined by $a^{\text {lat }}(t)=v(t)^{2} \kappa(s(t))$ so that the normalized steering input $u_{1}$ is no longer a control input to the vehicle. The state vector for the movement along a path reduces to $\tilde{x}=\left[\begin{array}{ll}s, & v\end{array}\right]^{T}$. Due to this abstraction, the longitudinal dynamics is monotone:

Definition 1 (Monotone dynamics; see [29]): The system dynamics is monotone with respect to the initial state $x(0) \in \mathcal{R}(0)$ and inputs $u(t) \in \mathcal{U}$ when the following property holds for the solution $\chi(t, x(0), u(\cdot))$ :

$$
\begin{aligned}
& \text { if } \forall i, j, t \geq 0: x_{i}(0) \leq \bar{x}_{i}(0), u_{j}(t) \leq \bar{u}_{j}(t) \text { then } \\
& \forall i, t \geq 0: \chi_{i}(t, x(0), u(\cdot)) \leq \chi_{i}(t, \bar{x}(0), \bar{u}(\cdot)) .
\end{aligned}
$$

A constructive methods to prove monotonicity is presented in [29], which returns monotonicity with respect to $\tilde{x}$ and $u_{2}$ ( $u_{1}$ is no longer an input). Thus, the upper bound on the path coordinate can be computed as follows: Start at the maximum initial position and velocity (within the set of possible initial 
states) and apply full possible acceleration. Obtaining the upper bound $\bar{u}_{2}(s)$ on the acceleration along a curved path considering $\mathrm{C} 4$ requires to solve an optimization problem for which a fast semi-analytical method exists [30]. The optimal solution is a bang-bang control, i.e. the input takes only the values $\bar{u}_{2} \in\{-1,1\}$ since the input is already normalized.

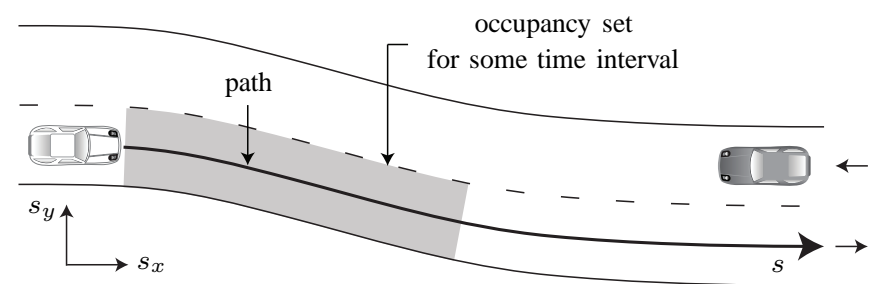

Fig. 3. Occupancy along road boundaries.

\section{B. Occupancy Towards Road Boundaries}

Computing the occupancy when the movement is not restricted along a path is much more challenging since there does not exist a single trajectory that defines the boundary for all times. This is demonstrated in Fig. 4, where simulations for different orientations of a vehiclefixed acceleration vector are plotted while the absolute value is always $a_{\max }$. The angle $\phi=90^{\circ}$ corresponds to a left turn without longitudinal acceleration, whereas $\phi=180^{\circ}$ corresponds to full braking without steering. It can be seen that for different times, solutions of different acceleration orientations $\left(\phi \in\left\{90^{\circ}, 110^{\circ}, 130^{\circ}\right\}\right)$ define the border of these 3 solutions. Note that even the union of positions for all acceleration directions is only a subset of the occupancy set, because the acceleration direction is allowed to be timevarying.

To simplify the analysis for the movement on the plane, we restrict ourselves to constraints $C 4$ and $C 5$ in this setting. This makes it possible to apply road-fixed acceleration inputs as shown in (1), resulting in a monotone dynamics, whereas the dynamics of vehicle-fixed acceleration inputs is not monotone. We first consider straight roads with uncertain initial states, where each state variable is bounded by an interval and the $x$-axis is aligned with the road direction. Due to monotonicity of (1), the lower left (11) and lower right (lr) occupancy boundary is obviously obtained by starting at

$$
\begin{aligned}
& x_{l l}(0)=\left[\begin{array}{llll}
\underline{s}_{x}(0), & \bar{s}_{y}(0), & \underline{v}_{x}(0), & \bar{v}_{y}(0)
\end{array}\right]^{T} \\
& x_{l r}(0)=\left[\begin{array}{llll}
\underline{s}_{x}(0), & \underline{s}_{y}(0), & \underline{v}_{x}(0), & \underline{v}_{y}(0)
\end{array}\right]^{T}
\end{aligned}
$$

where under- and overlines represent the lower and upper limits of initial states. This is indicated for uncertain initial positions in Fig. 2. Based on the worst-case initial states, we present three methods for computing the occupancy boundary for arbitrary time-varying acceleration inputs.

1) Method A (Exact Tire Friction Limit): We first consider the occupancy using the abstraction that only the absolute acceleration is limited (constraint $C 4$ ). In this setting, the occupancy of the vehicle can be described by circles with center $c(t)$ and radius $r(t)$ when the initial position and

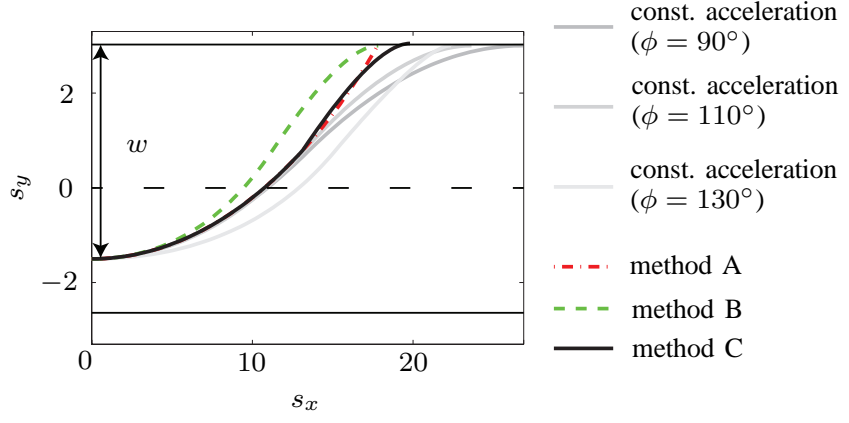

Fig. 4. Occupancy boundary for changing to the left lane.

velocity is known [5]:

$$
c(t)=\left[\begin{array}{l}
s_{x}(0) \\
s_{y}(0)
\end{array}\right]+\left[\begin{array}{l}
v_{x}(0) \\
v_{y}(0)
\end{array}\right] t, \quad r(t)=\frac{1}{2} a_{\max } t^{2} .
$$

In order to compute the occupancy for time intervals, one would have to unify infinitely many circles. In order to avoid this problem, we derive the boundary of all circles (see Fig. 5):

Proposition 2 (Boundary of Occupancy): Without loss of generality, we choose $s_{x}(0)=0, s_{y}(0)=0, v_{x}(0)=v_{0}$, and $v_{y}(0)=0$. The $x$ - and $y$-coordinate of the boundary are:

$b_{x}(t)=v_{0} t-\frac{a_{\max }^{2} t^{3}}{2 v_{0}}, \quad b_{y}(t)=\sqrt{\frac{1}{4} a_{\max }^{2} t^{4}-\left(\frac{a_{\max }^{2} t^{3}}{2 v_{0}}\right)^{2}}$.

Proof: To simplify the proof, the new variable $\tilde{b}_{x}(t)=$ $b_{x}(t)-v_{0} t$ is introduced. The possible $x$ - and $y$-positions of the two circles with radius $r(\cdot)$ at time $t$ and $t+\Delta t$ are:

$$
\begin{aligned}
\tilde{b}_{x}^{2}+b_{y}^{2} & =r^{2}(t), \\
\left(\tilde{b}_{x}-v_{0} \Delta t\right)^{2}+b_{y}^{2} & =r^{2}(t+\Delta t) .
\end{aligned}
$$

Inserting $b_{y}^{2}=r^{2}(t)-\tilde{b}_{x}^{2}$ from (2) into (3) and some rewriting results in the $x$-coordinate of their intersection:

$$
\tilde{b}_{x}=\frac{r^{2}(t)-r^{2}(t+\Delta t)}{2 v_{0} \Delta t}+\frac{1}{2} v_{0} \Delta t .
$$

Using $r(t)=\frac{1}{2} a_{\max } t^{2}$, we obtain after some calculations

$$
r^{2}(t)-r^{2}(t+\Delta t)=\left(-a_{\max }^{2} t^{3}+\mathcal{O}(\Delta t)\right) \Delta t,
$$

where $\mathcal{O}(\Delta t)$ includes linear and higher order terms of $\Delta t$. Inserting the above result into (4) and computing the limit for $\Delta t \rightarrow 0$ results in $\tilde{b}_{x}(t)$ and $b_{y}(t)$ due to (2).

The occupancy $O\left(t_{k}\right)$ for specific points in time $t_{k}$ as well as the left and right boundary are plotted in Fig. 5 for the initial velocity $v_{0}=20 \mathrm{~m} / \mathrm{s}$ and $a_{\max }=10 \mathrm{~m} / \mathrm{s}^{2}$. The occupancy for a time interval $\left[t_{k}, t_{k+1}\right]$ is bounded by the left and right boundaries from time $t_{k}$ to time $t_{k+1}$ and the circles at time $t_{k}$ as well as $t_{k+1}$. For implementation, a convex hull of both circles is used as an reasonable overapproximation (see gray area in Fig. 5). It is obvious that method A allows behaviors that result in driving backwards, which is resolved by setting $b_{x}(t)=b_{x}\left(t^{*}\right)$ after time $t^{*}=v_{0} / a_{\max }$ for which it is no longer ensured that the vehicle has not come to a stop. 


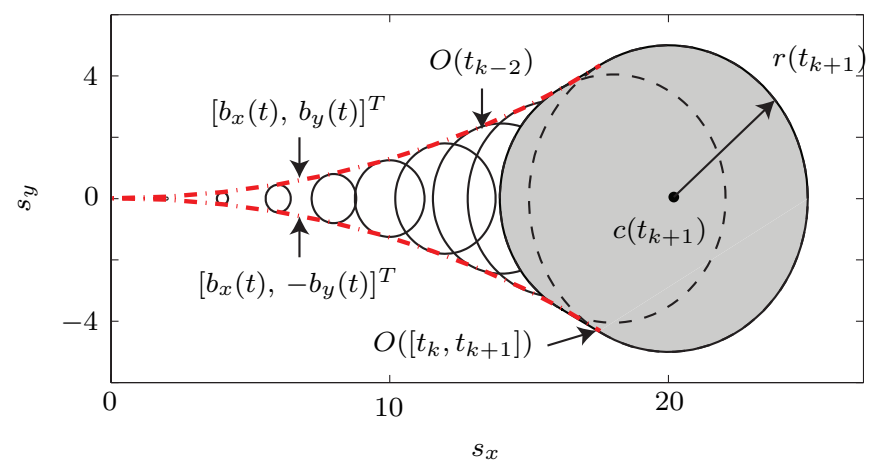

Fig. 5. Occupancy sets according to method A.
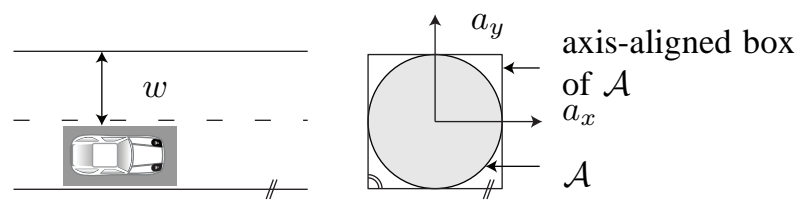

Fig. 6. Enclosing the acceleration set by an axis-aligned box.

2) Method B (Overapproximative Tire Friction Limit and Drivable Area Restriction): Method B focuses on forbidding behaviors that leave the road boundary (constraint $C 5$ ), but also considers an overapproximation of constraint $C 4$ on possible acceleration values. A simple solution that exactly considers constraint $C 4$ and $C 5$ is yet unknown. We first present the idea for a straight road and then generalize it to an arbitrarily curved road. Method B decouples the dynamics in $x$ - and $y$-direction by overapproximating the set of possible accelerations $\mathcal{A}=\left\{\left[a_{x}, a_{y}\right]^{T} \mid\left\|\left[a_{x}, a_{y}\right]^{T}\right\|_{2} \leq a_{\max }\right\}$ by an axis-aligned box, see Fig. 6. Since according to this simplification, the vehicle can fully accelerate in $y$-direction independently of the movement in $x$-direction, the fastest trajectory to reach the road boundary is to fully accelerate in $y$-direction until full opposite acceleration is required to stay within the road boundary.

Proposition 3 (Time for Switching Acceleration): The time $t_{s}$ for switching the acceleration direction for a distance $w$ to the road boundary (see Fig. 6) is

$$
t_{s}=\frac{\sqrt{a_{\max } w+\frac{1}{2} v_{0}^{2}}-v_{0}}{a_{\max }}
$$

for $\sqrt{a_{\max } w+\frac{1}{2} v_{0}^{2}}-v_{0} \geq 0$, otherwise leaving the road cannot be prevented.

Proof: The remaining distance $w-s_{y}\left(t_{s}\right)$ has to match the distance required to reach $v_{y}=0$ :

$$
w-s_{y}\left(t_{s}\right)=\frac{v_{y}^{2}\left(t_{s}\right)}{2 a_{\max }}
$$

where $s_{y}\left(t_{s}\right)=\frac{1}{2} a_{\max } t_{s}^{2}+v_{y}(0) t_{s}, v_{y}\left(t_{s}\right)=a_{\max } t_{s}+v_{y}(0)$. After inserting $s_{y}\left(t_{s}\right)$ and $v_{y}\left(t_{s}\right)$, one has to find the zeros of a quadratic function of $t_{s}$. The non-negative solution is the one of the proposition.

A lower and upper corner of the occupancy set are obtained by combining the movement in $y$-direction with full deceleration and acceleration in $x$-direction due to the independence assumption of this subsection. As for method A, we set $s_{x}(t)=s_{x}\left(t^{*}\right)$ after time $t^{*}=v_{0} / a_{\max }$ for which driving backwards is possible. The lower bound (in driving direction) is shown in Fig. 4. Note that for larger initial velocities than the $20 \mathrm{~m} / \mathrm{s}$ used for Fig. 4, method B is less overapproximative compared to method A.

3) Method C (Combining Method A and B): So far it has been shown that method $\mathrm{A}$ is exact in the first phase $\left(t \leq t_{s}\right)$ of the lane change, but performs worse than method B in the second phase $\left(t>t_{s}\right)$, see Fig. 4 . The natural extension is to combine both approaches by first using method A until time $t_{s}$ and then apply method B. We first keep the straight-road setting, which is later extended. The initial state $\tilde{x}\left(t_{s}\right)$ of the second phase is chosen as

$$
\tilde{x}\left(t_{s}\right)=\left[b_{x}\left(t_{s}\right), \quad b_{y}\left(t_{s}\right), \quad v_{0}-a_{\max } t_{s}, \quad \pm a_{\max } t_{s}\right]^{T} .
$$

The initial position is obviously the final position of the first phase, whereas the initial velocity requires some more explanation. There does not exist a single initial state $x\left(t_{s}\right)$ from which all solutions start that define the boundary of the occupancy for some time. However, due to monotonicity, method $B$ bounds the occupancy when choosing the lowest possible velocity in $x$-direction and the highest/lowest possible velocity in $y$-direction for the left/right bound. Note that the initial state $\tilde{x}\left(t_{s}\right)$ is not reachable, but provides a worstcase initial state for method $B$. The plots of method $\mathrm{C}$ in comparison with method A and B are shown in Fig. 4.

4) Arbitrarily Curved Roads: So far, method C has only been presented for straight roads. In order to use the method for arbitrarily curved roads, we first introduce the section of the road boundary between points $P_{1}$ and $P_{2}$ (see Fig. 7) within which the occupancy boundary is guaranteed to hit the road boundary. Point $P_{1}$ can be obtained by intersecting the solution of method $A$ with the road boundary. Point $P_{2}$ is obtained by computing a solution that touches the road boundary such that the velocity direction at $P_{2}$ is aligned with the tangent of the road boundary. Since this is a solution that does not leave the road boundary, it marks the other end of the potential intersection region. The solution ending in $P_{2}$ is computed using $a^{\text {lat }}(t)=\operatorname{sgn}(f(t)) a_{\max }, a^{\text {long }}(t)=$ 0 , where $f(t)$ is a function over time, so that the solution consists of two arcs with radius $\frac{v_{0}^{2}}{a_{\max }}$. It remains to find the time to switch the direction of the lateral acceleration. This is done iteratively using binary search: If the solution hits the road boundary, the switching time has to be reduced, if the road boundary is not hit, the time is enlarged. This is done until the solution hits the road boundary and the direction vector is within a user-defined cone of the tangent vector at $P_{2}$.

Once $P_{1}$ and $P_{2}$ are obtained, we compute a halfspace (see Fig. 7) that does not intersect the road boundary between $P_{1}$ and $P_{2}$, but is as close as possible to the road boundary. This is achieved by pushing the line connecting $P_{1}$ and $P_{2}$ outwards until the path does not intersect anymore. When the road segment between $P_{1}$ and $P_{2}$ is concave, the connecting line segment already defines the boundary of the halfspace. 
Given this halfspace, we introduce a road-fixed coordinate system whose $y$-axis is aligned with the normal vector of the halfspace. Since this specific orientation of the $y$-axis with respect to the road boundary is the only prerequisite for applying method $\mathrm{C}$, it can be identically applied as presented for straight roads. It is mentioned that the result is slightly more overapproximative for curved roads since the occupancy boundary touches the halfspace outside the road surface.

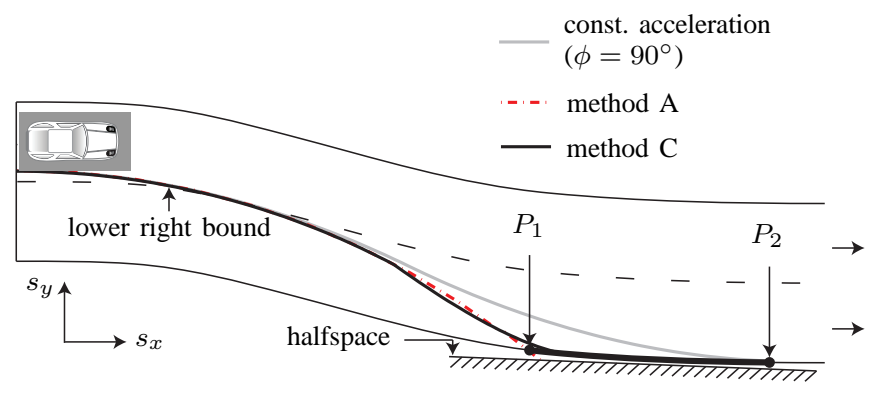

Fig. 7. Lower right boundary of the occupancy on a curved road.

\section{NumERICAL EXAMPLES}

This section presents two scenarios that demonstrate how the occupancy sets are integrated into the trajectory planning for potential emergency maneuvers. The trajectory planning is performed as described in [31] using rapidly exploring random trees (RRTs). Both scenarios use a straight road with two lanes for traffic in the same direction, which can be described by only specifying the lane width $w=3 \mathrm{~m}$. In the first scenario, the ego car follows vehicle V2, while another vehicle V1 occupies the neighboring lane. In this setting, the ego vehicle has only a chance to avoid a collision by braking, while an evasive maneuver is impossible. The parameters of this scenario are listed in Tab. I and the results of the occupancy set computation as well as the trajectory planning are illustrated in Fig. 8. The time-position plot shows the occupancy sets for small consecutive time intervals with a chosen time increment of $t_{k+1}-t_{k}=0.1 \mathrm{~s}$. Once the occupancy sets are computed over time, the trajectory planner computes partial trajectories (see gray lines in Fig. 8) as long as the occupancy of the ego vehicle does not intersect with any of the occupancy sets of other traffic participants or comes to a safe stop. The best collision-free emergency trajectory according to a specified cost function (here: jerk minimization) is plotted by a thick black line. As expected, the best collision-free trajectory is a braking maneuver in the current lane.

Similarly to the first scenario, the ego vehicle approaches a slower vehicle in the second scenario. But this time, the vehicle on the neighboring lane is positioned such that an evasive maneuver is possible, while a braking maneuver is no longer possible. Note that a braking maneuver would have been possible earlier, but since evasive maneuvers are a safe option, automatic braking has not been activated previously. The parameters of this scenario are listed in Tab. I and the

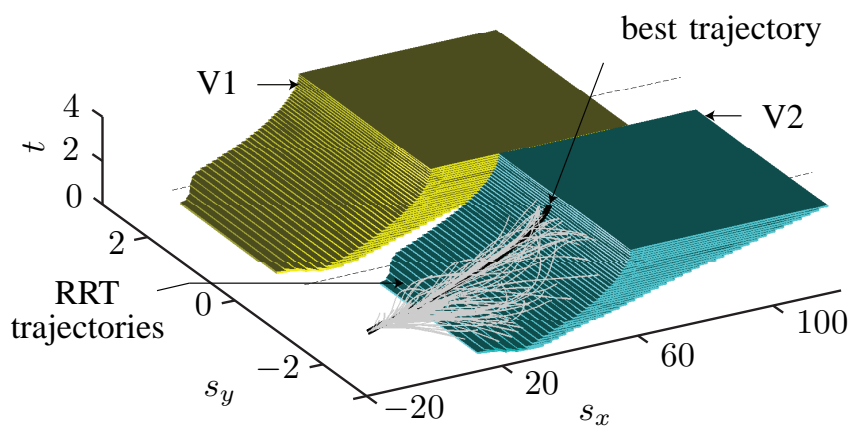

Fig. 8. Collision avoidance planning of scenario 1. The gray lines show partial trajectories of the RRT planner, the thick black line shows the best potential emergency trajectory, and the $3 \mathrm{D}$ regions show the occupancy sets over time.

results are presented as for the first scenario by a timeposition plot in Fig. 9. The best collision-free solution (thick black line) is as expected an evasive maneuver.

The RRT planner as well as the computation of the occupancy sets are implemented in MATLAB. The computation time for the occupancy sets are 0.019 seconds using an Intel i7-2620M CPU running at $2.7 \mathrm{Ghz}$. We could achieve the low computation times since we have a closed-form solution of method $\mathrm{C}$ and because there exists a piecewise closed-form solution of the longitudinal dynamics for the upper bound of the occupancy set as presented in [32, Prop. 1].

TABLE I

SCENARIO PARAMETERS.

\begin{tabular}{lccc}
\hline variable & ego & V1 & V2 \\
\hline \hline \multicolumn{4}{c}{ parameters of both scenarios } \\
\hline$a_{\max }\left[\mathrm{m} / \mathrm{s}^{2}\right]$ & - & 5 & 5 \\
$v_{\max }[\mathrm{m} / \mathrm{s}]$ & - & 30 & 30 \\
$v_{S}[\mathrm{~m} / \mathrm{s}]$ & - & 7 & 7 \\
\hline \multicolumn{5}{c}{ parameters of 1st scenario } \\
\hline$s_{x}(0)[\mathrm{m}]$ & 0 & {$[-2.5,2.5]$} & {$[17.5,22.5]$} \\
$s_{y}(0)[\mathrm{m}]$ & -1.5 & {$[0.3,2.7]$} & {$[-2.7,-0.3]$} \\
$v_{x}(0)[\mathrm{m} / \mathrm{s}]$ & 20 & {$[20,22]$} & {$[20,22]$} \\
$v_{y}(0)[\mathrm{m} / \mathrm{s}]$ & 0 & {$[-0.2,0.2]$} & {$[-0.2,0.2]$} \\
\hline \multicolumn{5}{c}{ parameters of 2 nd scenario } \\
\hline$s_{x}(0)[\mathrm{m}]$ & 0 & {$[47.5,52.5]$} & {$[17.5,22.5]$} \\
$s_{y}(0)[\mathrm{m}]$ & -1.5 & {$[-0.1,2.3]$} & {$[-2.7,-0.3]$} \\
$v_{x}(0)[\mathrm{m} / \mathrm{s}]$ & 20 & {$[25,27]$} & {$[15,17]$} \\
$v_{y}(0)[\mathrm{m} / \mathrm{s}]$ & 0 & {$[-0.2,0.2]$} & {$[-0.2,0.2]$} \\
\hline \multicolumn{5}{c}{}
\end{tabular}

\section{CONCLUSION}

We present a new approach for predicting the road occupancy of other traffic participants for collision avoidance systems. In order to guarantee safety under all possible behaviors of other traffic participants, a techniques for obtaining overapproximative occupancy sets rather than approximations is introduced. To ensure that all behaviors are considered, one has to choose large enough model uncertainties - this problem, however, also arises when simulations or stochastic methods are used. The presented set-based technique eliminates issues related to incomplete prediction for a given model. Thus, our approach is a building block 


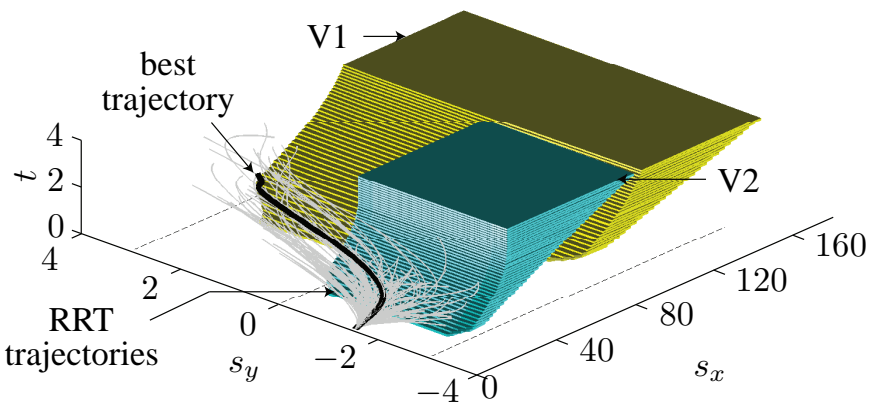

Fig. 9. Collision avoidance planning of scenario 2. The gray lines show partial trajectories of the RRT planner, the thick black line shows the best potential emergency trajectory, and the 3D regions show the occupancy sets over time.

for the formal verification of collision avoidance maneuvers. Another advantage is that the computation time is only a few hundredth of a second. In the future, we plan to add more restrictions, specifically concerning interaction with other vehicles. It is worth mentioning that unconsidered restrictions only result in larger occupancies, which does not affect safety, but triggers emergency maneuvers earlier than possible. This inherent safety property is one of the main strengths of the presented concept.

\section{REFERENCES}

[1] K. D. Kusano and H. C. Gabler, "Safety benefits of forward collision warning, brake assist, and autonomous braking systems in rear-end collisions," IEEE Transactions on Intelligent Transportation Systems, vol. 13, no. 4, pp. 1546-1555, 2012.

[2] S. M. LaValle, Planning Algorithms. Cambridge University Press, 2006.

[3] H. Täubig, U. Frese, C. Hertzberg, C. Lüth, S. Mohr, E. Vorobev, and D. Walter, "Guaranteeing functional safety: design for provability and computer-aided verification," Autonomous Robots, vol. 32, no. 3, pp. 303-331, 2012.

[4] J. van den Berg, D. Ferguson, and J. Kuffner, "Anytime path planning and replanning in dynamic environments," in Proc. of the International Conference on Robotics and Automation, 2006, pp. 2366-2371.

[5] C. Schmidt, F. Oechsle, and W. Branz, "Research on trajectory planning in emergency situations with multiple objects," in Proc. of the IEEE Intelligent Transportation Systems Conference, 2006, pp. 988-992.

[6] A. Wu and J. P. How, "Guaranteed infinite horizon avoidance of unpredictable, dynamically constrained obstacles," Autonomous Robots, vol. 32, no. 3, pp. 227-242, 2012.

[7] D. Greene, J. Liu, J. Reich, Y. Hirokawa, A. Shinagawa, H. Ito, and T. Mikami, "An efficient computational architecture for a collision early-warning system for vehicles, pedestrians, and bicyclists," IEEE Transactions on Intelligent Transportation Systems, vol. 12, no. 4, pp. 942-953, 2011.

[8] N. Kaempchen, B. Schiele, and K. Dietmayer, "Situation assessment of an autonomous emergency brake for arbitrary vehicle-to-vehicle collision scenarios," IEEE Transactions on Intelligent Transportation Systems, vol. 10, pp. 678-687, 2009.

[9] B. Vanholme, D. Gruyer, B. Lusetti, S. Glaser, and S. Mammar, "Highly automated driving on highways based on legal safety," IEEE Transactions on Intelligent Transportation Systems, vol. 14, no. 1, pp. 333-347, 2013.

[10] D. Ferguson, M. Darms, C. Urmson, and S. Kolski, "Detection, prediction, and avoidance of dynamic obstacles in urban environments," in Proc. of the IEEE Intelligent Vehicles Symposium, 2008, pp. 11491154.

[11] A. Eidehall, "Multi-target threat assessment for automotive applications," in Proc. of the 14th Int. IEEE Conference on Intelligent Transportation Systems, 2011, pp. 433-438.
[12] A. Barth and U. Franke, "Where will the oncoming vehicle be the next second?" in Proc. of the IEEE Intelligent Vehicles Symposium, 2008, pp. 1068-1073.

[13] M. Brännström, E. Coelingh, and J. Sjöberg, "Model-based threat assessment for avoiding arbitrary vehicle collisions," IEEE Transactions on Intelligent Transportation Systems, vol. 11, no. 3, pp. 658-669, 2010.

[14] S. Bonnin, F. Kummert, and J. Schmüdderich, "A generic concept of a system for predicting driving behaviors," in Proc. of the 15th Int. IEEE Conference on Intelligent Transportation Systems, 2012, pp. $1803-1808$

[15] I. Dagli and D. Reichardt, "Motivation-based approach to behavior prediction," in Proc. of the Intelligent Vehicles Symposium, 2002, pp. 227-233.

[16] H. Jula, E. B. Kosmatopoulos, and P. A. Ioannou, "Collision avoidance analysis for lane changing and merging," IEEE Transactions on Vehicular Technology, vol. 49, pp. 2295-2308, 2000.

[17] A. Kanaris, E. B. Kosmatopoulos, and P. A. Ioannou, "Strategies and spacing requirements for lane changing and merging in automated highway systems," IEEE Transactions on Vehicular Technology, vol. 50, pp. 1568-1581, 2001.

[18] Y. Nishiwaki, C. Miyajima, H. Kitaoka, and K. Takeda, "Stochastic modeling of vehicle trajectory during lane-changing," in Proc. of the IEEE International Conference on Acoustics, Speech and Signal Processing, 2009, pp. 1377-1380.

[19] G. Xu, L. Liu, Y. Ou, and Z. Song, "Dynamic modeling of driver control strategy of lane-change behavior and trajectory planning for collision prediction," IEEE Transactions on Intelligent Transportation Systems, vol. 13, no. 3, pp. 1138-1155, 2012.

[20] A. Eidehall and L. Petersson, "Statistical threat assessment for general road scenes using Monte Carlo sampling," IEEE Transactions on Intelligent Transportation Systems, vol. 9, pp. 137-147, 2008.

[21] A. E. Broadhurst, S. Baker, and T. Kanade, "Monte Carlo road safety reasoning," in Proc. of the IEEE Intelligent Vehicles Symposium, 2005, pp. 319-324.

[22] M. Althoff, O. Stursberg, and M. Buss, "Model-based probabilistic collision detection in autonomous driving," IEEE Transactions on Intelligent Transportation Systems, vol. 10, pp. 299 - 310, 2009.

[23] J. Hardy and M. Campbell, "Contingency planning over probabilistic hybrid obstacle predictions for autonomous road vehicles," in Proc. of the IEEE/RSJ International Conference on Intelligent Robots and Systems, 2010, pp. 2237-2242.

[24] T. Gindele, S. Brechtel, J. Schröder, and R. Dillmann, "Bayesian occupancy grid filter for dynamic environments using prior map knowledge," in Proc. of the IEEE Intelligent Vehicles Symposium, 2009, pp. 669-676.

[25] J. Overstreet and F. Khorrami, "Multi-ordered short-range mover prediction models for tracking and avoidance," in Proc. of the 51st IEEE Conference on Decision and Control, 2012, pp. 1770-1775.

[26] D. Hess, M. Althoff, and T. Sattel, "Comparison of trajectory tracking controllers for emergency situations," in Proc. of the 16th IEEE Conference on Intelligent Transportation Systems, 2013.

[27] M. Althoff, "Reachability analysis and its application to the safety assessment of autonomous cars," Dissertation, Technische Universität München, 2010, http://nbnresolving.de/urn/resolver.pl?urn:nbn:de:bvb:91-diss-20100715963752-1-4.

[28] M. Althoff and B. H. Krogh, "Zonotope bundles for the efficient computation of reachable sets," in Proc. of the 50th IEEE Conference on Decision and Control, 2011, pp. 6814-6821.

[29] D. Angeli and E. D. Sontag, "Monotone control systems," IEEE Transactions on Automatic Control, vol. 48, no. 10, pp. 1684-1698, 2003.

[30] E. Velenis and P. Tsiotras, "Optimal velocity profile generation for given acceleration limits: theoretical analysis," in Proc. of the American Control Conference, 2005, pp. 1478 - 1483.

[31] D. Heß, "Evaluation of motion planning strategies for autonomous cars," Master's thesis, Universität Paderborn, 2009.

[32] M. Althoff and A. Mergel, "Comparison of Markov chain abstraction and Monte Carlo simulation for the safety assessment of autonomous cars," IEEE Transactions on Intelligent Transportation Systems, vol. 12, no. 4, pp. 1237-1247, 2011. 